feeling of justice for the animal. He quickly inspires $l_{0.1}$ to 0.5 of carbon, we may refine the exterior of the
his horses with respect and confidence-for he rarely fragments by heating them for some hous uses the whip or force-and seldom or never with fear. Therein lies the secret of his great success.

The result of his breaking and training is shown in a pleasing manner in a feat that reminds one of the attraction as one of the "Hungarian Pictures" at th
recent Millennium Exposition. We refer to the feat illustrated in the accompanying engraving, in which by Fraulein Motzelt on a moving roller 79 inches in diameter. By a light, elegant spring the horse reaches
the top of the roller, which is provided with slats an inch apart and running parallel with the long axis of facilitate the rolling movement.

facilitate the rolling movement.
Once on the roller Hengst rolls it along by stepping orward slowly, until it reaches the edge of the track then he jumps down, rises on his hind legs, and in this
position crosses the track and leaves the theater of his posiumphs his rider clinging to him as if cast in the
saddle. During the ride on the moving roller the horse and rider present a fine appearance; the noble beast is so quiet that he reminds one of a monumental statue, is wonderful that by pure force of will his master has
made him perform one of the most difficult tasks that could be set for any horse, eve

\section{PREPARATION AND PROPERTIES OF}

By Henri Moissan, in Comptes Rendus.

In a paper published on Febrnary 20,1893 , we hav not reducible by carbon, can yield metallic uranium in presence of this substance at the high temperature of
my electric furnace. We have since demonstrated the

and carbon, $\mathrm{C}_{s} \mathrm{U}_{2 .}$.
Whall now give a more complete study of this Imt is known that metallic uranium was first prepared by Peligot by reducing uranium chloride with potassium in a platinum crucible. By this procedure we obtain a gray powder, in the midst of which are found
some small metallic globules. tion, and in 1886 Zimmermann resumed the study of uranium, and obtained the metal by reducing uranium chloride with sodium. The isolated metallic grobules in this preparation were few in unsuber. Their fusion the alkaline metal upon the chloride.

ate in a platinum crucible, the uranium is al ways soiled with this metal. In Zimmermann's preparation the small quantity of sodium.

Moreover, whatever is the method employed, all these uraniums in powder contain nitrogen and often oxygen. strong affinity for gaseous nitrogen, hitherto unknown. might be more advantageously employed by means of a Preparation of Double Uranium and Sodium Chloride, $\mathrm{UCl}_{4}, 2 \mathrm{NaCl}$. - When we pass a current of vapor of uranium chloride over sodium chloride we obtain a double chloride, which on cooling congeals to a crystalline mass of an apple green color, melting at $390^{\circ}$, by alcohol.

This preparation is very easily made in a tube of Bohe mian glass, forming at one end uranium chloride by ing this chloride to pass over fragments of sodium chloride placed at the other end and heated to dull redness. all the vapor of uranium chloride, and then the mass melts rapidly

We know that uranium chloride, $\mathrm{UCl}_{4}$, is greedy of moisture, fumes in the air, and is not easily manageable.
On the contrary, the crystalline double chloride is much vess hygroscopic and more stable. If fused, it yields a vapors.
Retuction of this Double Chloride by Alkaline Metals.-The reduction has been effected in a very thick iron cylinder, closed with a screw stopper. It is
charged with alternate layers of 300 grms. double hloride and 100 grms. sodium recently cut.

The apparatus being closed, it is placed in a very brisk wood fire, when it is heated for twenty-five
minutes. The heat disengaged by the reaction is so intense as to raise the block of iron to a cherry red heat in a few instants. When cold, the cylinder is opened, at $96^{\circ}$ to remove the excess of sodium, then rapidly washed with water which has been boiled and allowed to cool, then exhausted with alcohol and lastly with ether. We have indicated in a former paper on uranium carfied. The uranium having been brought to the state of green oxide $\left(\mathrm{U}_{3} \mathrm{O}_{8}\right)$, is intimately mixed with suga
charcoal in fine powder in the following proportions : Uranium oxide 500 grms.

about $500 \mathrm{grms}$. of this mixture are placed in a coke crucible and submitted in the electric furnace for seven volts. We thus obtain a fused ingot of about 350 grms.
The metal thus prepared if the heating has been well The metal thus prepared, if the heating has been well conducted, contains very little carbon, and sometimes
even not a trace. On the contrary, we may find in it a mall quantity of oxide, which then wrnishes a "burnt metal, the physical properties of which are notably
modified. If the duration of the heat is too long, the metal is readily carbized, and we obtain a cast metal action of nitrogen, it is better to make these experi-

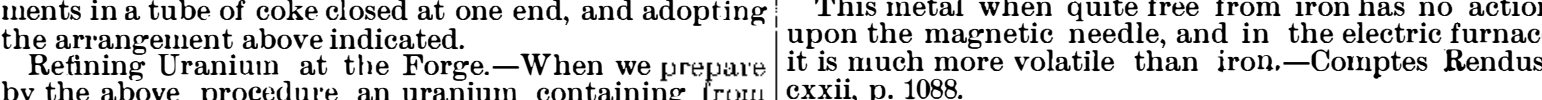
Refining Uranium at the Forge.- When we prepare
by the above procedure an uranium containing Irom
cxxii, p. 1088.

ped with green uranium oxide. To realize this experiment we must place the crucible containing the
uranium oxide on the metal in the middle of another rucible filled with titaniferous carbon finely pulverized metal coated with nitride.
might produce a yellow
. Preparation of Metallic Uranium by Electrolysis.-
The double uranium chloride above described is electrolyzed with the greatest ease. It yields at the nega-
tive pole a sponge of uranium containing frequently sull crystals of this metal. To have regular working
it is sufficient to have at the terminals a difference of potential of 8 to 10 volts. We have generally used a in fusion by the thermic action of the current itself. pure charcoal, and the chloride is placed in a cylinpure charcoal, and the chloride is placed in a cylinlectrodes and to a porcelain, giving passage to the two his tube enables us to introduce above the melted salt a current of hydrogen very dry and very free from After complete cooling, the contents of the crucible are taken up with ice water, and washed rapidly with
alcohol ; for uranium, if finely divided, decomposes water at the ordinary temperature.
This uranium is crystalline, and certain parts near This uranium is crystalline, and certain parts near
the electrode appear even' in crystals measuring $1 \mathrm{~mm}$. If we employ an iron electrode, we obtain alloys of iron and uraniuni of a silvery white, which may be
easily filed and which have a very fine grain. color is absolutely white, less bluish than that of iron, though it takes a similar polish. If the metal has a yellow tint, we may always suspect the presence of
nitrogen. To its specific gravity we shall return below. Pure uranium may be easily filed; it does not seratch
glass. It may be slightly carbized if heated in a crucilass. It may le slightly carbized if heated
ble lined with carbon, and may be tempered.

Uranium is not magnetic when quite free from iron. ile than iron.

Chemical Properties. - Uranium in fine powder, as prepared by electrolysis, takes fire in fluorine, burns color Chlorine attacks it at the temperature of $180^{\circ}$,
and bromine at $210^{\circ}$, both with incandescence. The ame reaction is produced in the vapor of iodine about $260^{\circ}$ with formation of uranium iodide. All these re-
actions are complete. The metal obtained by Zimmerunn was not attacked by the vapor of iodine, and in urrent of chlorine it underwent a lineth

Hydrochloric acid gas attacks it with incandescence
at a dull red heat, yielding a stable chloride which proa dull red heat, yielding a stable chloride which pro-
duces a green solution in water. Hydriodic acid attacks it near a red heat.

Uranium in fine powder burns in pure oxygen at $170^{\circ}$ and upward, producing a very dark green oxide. Sulwhich is slowly attacked by hydrochloric acid, yielding ndrogen sulphide. With selenium it combines with
As we have remarked above, uranium combines with As we have remarked above, uranium combines with
nitrogen with the greatest ease. Fragments of the netal heated to $1,000^{\circ}$ in a current of nitrogen became
covered with a yellow layer of nitride. Uranium in powered with a yellow layer of nitride.
podnes reacts with a gaseous ammonia above dul dul
redness, but without incandescence, producing an escape of hyt rogen and leaving a black crystalline powPure uranium in very fine powder decomposes wate
Pung slowly at ordinary temperatures and more rapidly at $100^{\circ}$. This property approaches it to iron, since accordMelteduranium in contact with water is covered with layer of oxide; this action is strikingly accelerated by the presence of carbonic acid.

Analysis. - In all these researches the uranium has and separated and determined in the form
and the carbon wed as carbon dioxide.

Double uranium-so
he following figures

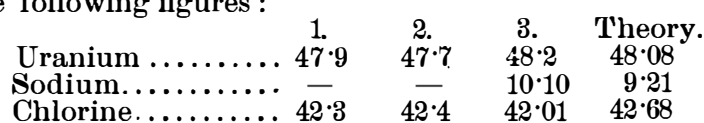

Metallic uranium prepared by sodium gave :

Uranium................. $99 \cdot 40 \quad 99 \cdot 28$

The specimen always contained traces of the alkaline

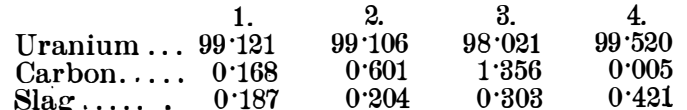

Uranium obtained by electrolysis :

Uranium.

Conclusions.-Metallic uranium may be easily ob-
ained either by decomposing the double uranium and sodjum fluoride with sodium or by the electrolysis of oxide with carbon in the electric furnace. These thre Uranium may

metal has properties approaching those of iron. It can me filed, carbized, tempered, and oxidized in the same
nanner. Its tendency to combine with oxygen is reater than that of iron : in fine powder it decompo:es greater than that of iron ; in fine powder it decompo:es
water slowly in the cold ; its reaction with the hydracids is also more energetic than that of iron. It has a more powerful affinity for nitrogen, and without special preautions the product obtained

This metal when quite free from iron has no action much more volatile than iron.-Comptes Rendus, the same compound, or preferably by reducing uranium

SELECTED FORMUL丑

Salt Water for Aquaria.-A satisfactory imitation salt water for use in aquaria in which sea fish are to e preserved may be made by dissolving in 18 gallons
f pure well or river water 1.25 grammes of sodiums
hloride, 90 grammes of magnesium sulphate, 22.5 grammes of potassium sulphate and 185 grammes of if the solution is allowed to stand in the open air in a cool place for, say, two or three weeks. A few sea
lgæ, such as grow on stones on the seashore, should As soon as the glass walls of the container
how small microscopic green spots, the water is in a show small microscopic green spots, the water is in a
condition to use. It is advisable to add some kind of ea weed, the genus Ulva being particularly useful, as .
tion.-American Druggist.

Paste for Pads. -

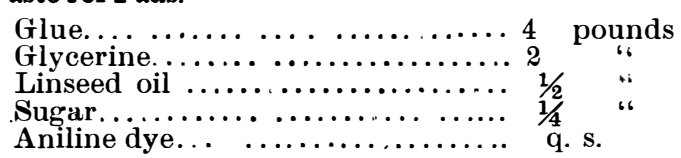

The flue is softened by soaking it in a little cold glycerine by aid of heat over a water bath. To this the dye is added, after which the oil is well stirred in. is used hot. Another composition
milar nature is prepared as follows.
Glue.........
Glucose sirup, about.
1 pound
4 ounces

Give the compositions an hour or more in which to dry or setbefore cutting or handling the pads.-Phar-

To Restore Crape.-Black crape may be freshened and made to look almost equal to new if treated in the following way : Lay over the ironing table a piece of black
cambric or cloth of any kind, and pin the piece of crape smoothly through to the blanket, stretching it out to out of water and lay it over the crape, patting it down with the palm of the hand. Now take hot flatirons and pass them over the wet cloth, letting them just When the cloth has become dry from the heat of the iron remove it, but let the crape remain pinned down until all the moisture has evaporated and
it is perfectly dry. The crape will now feel and look making sure that the part redressed comes under the Inaking sure that the part redressed comes und
edge of the wet cloth.-Lady Charlotte in London.

Koumyss.-In addition to the formula given in the submitted
Fresh milk
Brown sug
.12 ounces
Compressed yeast...
$21 / 2$ drachms
Milk sugar
3 arachm

Dissolve the milk sugar in the water, add to the milk,
rub the yeast and brown sugar down in a mortar with a little of the mixture, then strain into the other porbottles being frequently used, and the corks should fit very tightly ; in fact, it is almost necessary to use a properly fixed it should be wired down. Many failures the result because the corks did not fit properly, formed and left a worthless preparation. It is furthe necessary to keep the preparation at a moderate temperature, and to insure the article being properly fin-
ished, the bottles are to be gently shaken each day for about ten minutes to prevent the clotting of casein. around the bottle during the shaking process, as the amount of gas generated is great, and should the botSome few days or contain a flaw it may give way. into the acid stage, and when this has taken place the preparation is much thicker. It is now in the proper

To Determine the Presence of Alum in Bread.-Von der Planken (Annal de Phar. Lourain) recommends that 10 to 20 grammes of bread be mixed with water to
form a paste; to this is added some sodium chloride free from alkali, 10 drops of freshly prepared tincture of Calcium carbonate. 5 grammes of freshly precipitated gether, and then washed into a beaker, adding water pernatant fluid,will assume a grayish to deep blue colo violet color is produced.

\section{Preventive Lotion Against Insect Bites.}

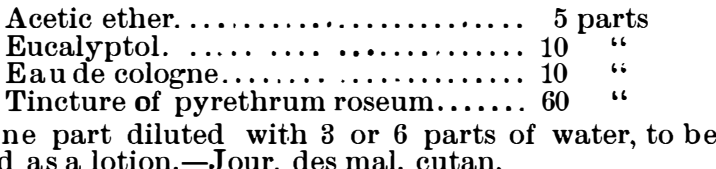

One part diluted with 3 or 6 parts
used as a lotion.-Jour. des mal. cutan.

To Paste Labels on Cork. - The following formulas Guin tragacanth

Dissolve in water, 1 pint; strain, and add thymol, 14 grains, suspended in glycerine, 4 ounces; finally add

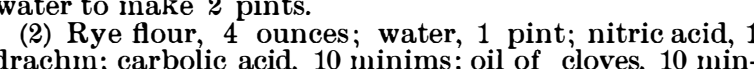
drachm; carbolic acid, 10 minims: oil of cloves, 10 minms; glycerine, 1 ounce. Mix the flour and water, strain
through cheese cloth, and add the nitric acid. Apply hrough cheese cloth, and add the nitric acid. Apply
heat until suitably thickened, and add the other in gredients when cooling. This paste is suitable for al-
most any kind of labels, and it will adhere to almost anything.

Stomatal.-This is a dentifrice which, according to of terpinol, 42 of water, 45 of alcohol, 2 of soap, 5 of
thents glycerine, and 2 of aromatics. 